INRA Prod. Anim., 2017, 30 (3), 219-228

\title{
Co-localisation des différentes productions animales en Europe : l'exception française ?
}

\author{
C. GAIGNÉ, E. LETORT
}

UMR SMART, INRA, Agrocampus Ouest, 35000, Rennes, France

Courriel : elodie.letort@inra.fr

\section{Certains territoires européens comme la Bretagne et la Lombardie concentrent plusieurs} productions animales tandis que des pays ont tendance à spécialiser leurs territoires dans une production (le lait en Allemagne, le porc en Espagne et au Danemark). Malgré a priori de faibles gains associés à cette co-agglomération de productions animales à l'échelle européenne, les modalités d'application des politiques agricoles et environnementales, en France notamment, semblent agir comme des freins au processus de mono-spécialisation des territoires d'élevage dans les filières avicoles, laitières et porcines.

Il est désormais bien établi que les productions animales, porcs, volailles et lait sont fortement concentrées dans quelques territoires au sein de l'Europe (Chatellier et Gaigné 2012, Gaigné et al 2012, Mosnier et Wieck 2012, Roguet et al 2015). Les mécanismes à l'œuvre dans ce processus ont fait l'objet de nombreux travaux d'économistes et de géographes. Les dotations naturelles et les modalités de l'intervention publique ne sont pas les seuls facteurs explicatifs de ce processus (Fujita et Thisse 2002). Depuis Cronon (1991), on souligne également le rôle important des économies d'agglomération, désignant l'ensemble des gains que retirent les producteurs liés à la proximité géographique avec d'autres producteurs d'une même filière. Pour éviter les coûts et les pertes de temps liés à l'éloignement, les producteurs sont incités à être situé à proximité de leurs transformateurs (industries d'aval) et de leurs fournisseurs (industries d'amont). Les éleveurs peuvent bénéficier d'avantages économiques liés à la simple proximité géographique entre producteurs d'une même filière. L'implantation dans un même territoire d'éleveurs favorise non seulement la circulation des informations et des innovations mais aussi le partage d'une main-d'œuvre et d'infrastructures spécifiques à une filière. De nombreuses études empiriques confirment l'existence de ces économies d'agglomération au sein des filières animales (Isik (2004) pour la filière lait, Roe et al (2002) et Gaigné et al (2012) pour la filière porcine). En contrepartie, cette concentration s'accompagne d'une forte concurrence pour l'accès aux facteurs de production, notamment au foncier et au travail, et a renforcé l'impact négatif des activités d'élevage sur l'environnement. Les normes environnementales fixant un seuil maximal d'azote ou de phosphore d'origine organique par hectare comme celles liées à la directive Nitrates n'ont pas vraiment freiné la concentration géographique des productions porcines et avicoles dans les territoires spécialisés en productions animales. Selon Gaigné et Ben Arfa (2011), les politiques environnementales ont parfois même pu accentuer la concentration des productions animales, en encourageant le recours à des solutions technologiques, comme le traitement du lisier.

Si la concentration spatiale au sein d'une même filière animale et les différents pertes et gains associés sont relativement bien identifiés, ceux associés à la présence de différentes productions animales au sein d'un même territoire (ce que l'on nomme par " co-localisation » ou " co-spécialisation ») sont rarement explicités. Dans les faits, certains territoires concentrent en même temps deux voire trois types de productions animales. Une analyse de la localisation des productions animales dans l'Union Européenne (UE) réalisée par Roguet et al (2015) a mis en évidence le fait que les productions de granivores (porcs et volailles) ont tendance à se localiser dans les mêmes zones géographiques. C'est le cas de la Catalogne en Espagne, des Flandres en Belgique et de la Basse-Saxe en Allemagne (tableau 1). Il existe évidemment d'autres types d'associations fréquentes comme les productions de lait et de viande bovine, et d'autres moins fréquentes comme les productions de porc et de lait. Quelques cas de figures sont particulièrement marquants dans la mesure où des régions accueillent massivement trois différentes filières animales. C'est le cas de la Bretagne qui concentre $56 \%$ des porcs, $33 \%$ des volailles et $20 \%$ des vaches laitières de l'hexagone sur seulement $6 \%$ de la SAU du pays. De même, la Lombardie, région du nord de l'Italie, concentre $30 \%$ des vaches laitières, $51 \%$ des porcs et $17 \%$ des volailles du pays pour seulement $8 \%$ de la SAU.

À travers ces exemples, les régions européennes productrices d'animaux se distinguent par le nombre de spécialisation (une unique ou plusieurs filières animales) et par la nature des combinaisons de spécialisation (différents types d'association de filières animales). Cet article est organisé comme suit. En premier lieu, nous présentons notre indicateur statistique pour mesurer la spécialisation des territoires. Dans une deuxième partie, nous proposons de réaliser un état des lieux de la co-localisation des différentes filières animales au sein des territoires européens, en identifiant notamment les associations de types de cheptel les plus récurrentes. La troisième partie discute du lien entre la nature des spécialisations et les densités animales à l'aide d'une analyse économétrique. Bien que ces analyses descriptives ne visent pas à expliquer le comportement économique sous-jacent à cette répartition spatiale des activités animales, nous chercherons à identifier des pistes pour expliquer les phénomènes de co-localisation des filières animales observés dans l'UE.

Les enjeux sont importants. Si les gains à l'agglomération au sein des différentes 
Tableau 1. Part des cheptels et part de la Surface Agricole Utile (SAU) dans des Régions de l'UE-28 exprimée en \% de leur pays respectif.

(Source : Eurostat 2010, traitement des auteurs).

\begin{tabular}{|l|c|c|c|c|}
\hline \multicolumn{1}{|c|}{ Régions } & \% Porcs & \% Volailles & \% Vaches Laitières & \% SAU \\
\hline Bavière (Allemagne) & 13 & 9 & $\mathbf{3 0}$ & 18 \\
\hline Sud-Est (Irlande) & 19 & 10 & $\mathbf{3 4}$ & 18 \\
\hline Basse-Saxe (Allemagne) & $\mathbf{3 1}$ & $\mathbf{3 9}$ & 18 & 15 \\
\hline Flandres Occ. (Belgique) & $\mathbf{5 2}$ & $\mathbf{3 3}$ & 17 & 15 \\
\hline Nord-Brabant (Pays-Bas) & $\mathbf{3 7}$ & $\mathbf{2 0}$ & 9 & 8 \\
\hline Catalogne (Espagne) & $\mathbf{2 8}$ & $\mathbf{2 7}$ & 9 & 5 \\
\hline Lombardie (Italie) & $\mathbf{5 0}$ & $\mathbf{1 7}$ & $\mathbf{3 0}$ & 8 \\
\hline Bretagne (France) & $\mathbf{5 6}$ & $\mathbf{3 3}$ & $\mathbf{2 0}$ & 6 \\
\hline
\end{tabular}

filières animales sont substantiels tandis que les gains liés aux interactions entre elles sont faibles, la séparation géographique de ces différentes filières peut apparaître souhaitable. Dans ce cas, les autorités publiques peuvent mener une politique d'aménagement du territoire incitant les territoires à mieux répartir leurs productions animales de manière à réduire les impacts négatifs sur l'environnement tout en maintenant le niveau de compétitivité de chaque filière. En revanche, si les avantages à la co-agglomération de différentes filières sont importantes, il est souhaitable d'identifier les combinaisons de filières (lait/ porc ; lait/viande bovine ; porc/volaille) procurant le plus d'intérêts économiques au niveau des territoires.

\section{1 / Mesure de la co-spéciali- sation des territoires}

Bien que la question de la mesure de la spécialisation ait fait l'objet de nombreuses recherches, il n'apparaît guère de consensus en ce qui concerne l'indicateur statistique le plus pertinent. Il est toutefois reconnu que la notion de spécialisation d'un territoire est à distinguer de la notion de concentration spatiale. Bien que les mesures utilisées pour décrire ces notions s'appuient sur la répartition des activités entre des zones géographiques, la concentration spatiale est une mesure spécifique à un type de production tandis que la spécialisation renvoie à un couple type de production/territoire. La manière dont les UGB ${ }^{1}$ se répartissent entre les territoires renvoie à la notion de concentration géographique d'une filière animale ${ }^{2}$. La spécialisation d'un territoire mesure la sur- (ou sous-) représentation d'un type de production dans un territoire par rapport au profil moyen du pays. C'est donc ce dernier type de mesure qui nous intéresse. Notre analyse de la spécialisation des territoires s'appuie sur un indice de spécialisation proche d'un indice de Gini relatif. Plutôt que d'analyser la spécialisation absolue d'un territoire, il est préférable d'étudier sa spécialisation relative en prenant comme référence le poids relatif des productions animales dans le pays. Par exemple, un territoire avec la même quantité d'UGB de vaches laitières et de volailles, ne signifie pas que le niveau de spécialisation dans les deux types de cheptel est le même pour ce territoire si ces dernières sont beaucoup moins nombreuses que les premières au niveau national. Au contraire, dans ce cas, ce territoire est davantage spécialisé dans les volailles. Le niveau de spécialisation d'un territoire dans une activité animale est donc indépendant de son niveau de production animale en valeur absolue. Par contre, ce mode de calcul peut impliquer qu'un territoire soit considéré comme spécialisé dans une production animale alors que la quantité absolue d'UGB de l'espèce animale sur ce territoire est inférieure à celle sur un territoire considéré comme non spécialisé. Par exemple, dans un pays où les porcs représentent $30 \%$ des UGB, un territoire avec seulement 9 UGB porcs et 1 UGB vache aura un indice de spécialisation mathématiquement très élevé en porc (égale à 3 , avec notre indicateur présenté plus bas). Nous tiendrons compte de cette limite dans nos analyses.

Nous chercherons dans un premier temps à mesurer si un territoire est spécialisé ou non dans une des trois productions animales identifiées en comparant le poids de cette activité animale dans ce territoire au poids de cette activité dans le pays. Ensuite, nous caractérisons tous les territoires de chaque pays de l'UE par leur nombre de spécialisation dans les différentes productions animales de manière à illustrer les phénomènes de co-localisation de différentes productions animales au sein d'un même territoire.

Pour mesurer si un territoire d'un pays donné est spécialisé dans une production donnée ou s'il accueille différentes espèces, nous avons calculé un indice de spécialisation par espèce $k$ et par territoire $r$ :

$$
S_{r}^{k}=\frac{U G B_{r}^{k} / \sum_{k} U G B_{r}^{k}}{\sum_{r} U G B_{r}^{k} / \sum_{r} \Sigma_{k} U G B_{r}^{k}}
$$

avec $k=($ vache laitière ; vache allaitante; porc ; volaille; autres espèces animales). Ainsi, mesure le nombre d'UGB de l'espèce $k$ dans le territoire $r$ pour chaque pays tandis que est la somme des UGB dans le territoire $r$, est la somme des UGB de type $k$ dans un pays et est la somme totale d'UGB dans un pays. Si $\mathrm{S}^{\wedge} k r>1$, le cheptel de type $k$ est surreprésenté dans le territoire $r$. Nous considérons alors que le territoire $r$ est spécialisé dans l'activité $i$. Le tableau 2 présente les différentes valeurs possibles prises par l'indicateur.

Pour chaque territoire au sein de chaque pays, nous déterminons ensuite le nombre de spécialisation dans les 4 principales productions animales, les vaches laitières, les vaches allaitantes, les porcs et les volailles. Chaque territoire est ainsi caractérisé par $0,1,2,3$ ou 4 spécialisations et par la nature de sa co-spécialisation (vaches laitières et allaitantes, porcs et volailles...). La catégorie correspondant aux autres espèces animales (les autres bovins, les ovins, les caprins et les équins) n'est pas directement analysée. Une analyse sur 8 productions animales en différentiant les autres bovins, les ovins, les caprins et les équins com-

\footnotetext{
${ }^{1}$ L’Unité de Gros Bétail (UGB) est une unité de référence permettant d'agréger le bétail de différentes espèces et de différents âges en utilisant des coefficients spécifiques établis initialement sur la base des besoins nutritionnels ou alimentaires de chaque type d'animal. Il existe un tableau de correspondance pour chaque espèce animale. Par exemple : vache laitière (1 UGB), vache allaitante ( $0,8 \mathrm{UGB})$, bovin entre un et deux ans $(0,7 \mathrm{UGB})$, truie reproductrice $(0,5)$, poulet de chair $(0,007)$, etc.

${ }^{2}$ La concentration spatiale des activités animales sera étudiée à partir des indices d'Herdinfhal, de Gini, ou encore d'Ellison et Glaeser.
} 
Tableau 2. Différentes valeurs prises par l'indice de spécialisation.

\begin{tabular}{|l|l|l|l|}
\hline \multicolumn{1}{|c|}{ Indice = 0 } & \multicolumn{1}{c|}{$\mathbf{0 < \text { Indice < 1 }}$} & \multicolumn{1}{c|}{ Indice = 1 } & \multicolumn{1}{c|}{ Indice > 1 } \\
\hline$\frac{U G B_{r}^{k}}{\sum_{k} U G B_{r}^{k}}=0$ & $\frac{U G B_{r}^{k}}{\sum_{k} U G B_{r}^{k}}<\frac{\sum_{r} U G B_{r}^{k}}{\sum_{r} \sum_{k} U G B_{r}^{k}}$ & $\frac{U G B_{r}^{k}}{\sum_{k} U G B_{r}^{k}}=\frac{\sum_{r} U G B_{r}^{k}}{\sum_{r} \sum_{k} U G B_{r}^{k}}$ & $\frac{U G B_{r}^{k}}{\sum_{k} U G B_{r}^{k}}>\frac{\sum_{r} U G B_{r}^{k}}{\sum_{r} \sum_{k} U G B_{r}^{k}}$ \\
\hline $\begin{array}{l}\text { L'activité } k \text { n'est pas } \\
\text { présente dans le } \\
\text { territoire } r .\end{array}$ & $\begin{array}{l}\text { L'activité } k \text { a moins de } \\
\text { poids dans les productions } \\
\text { animales du territoire } r \text { que } \\
\text { dans le pays. }\end{array}$ & $\begin{array}{l}\text { Le poids de l'activité } k \\
\text { dans les activités animales } \\
\text { du territoire } r \text { est identique } \\
\text { à leur poids } \\
\text { dans l'ensemble du pays. }\end{array}$ & $\begin{array}{l}\text { L'activité } k \text { a plus de poids } \\
\text { dans les productions } \\
\text { animales du territoire } r \text { que } \\
\text { dans le pays. }\end{array}$ \\
\hline $\begin{array}{l}\text { Le territoire } r \text { n'est } \\
\text { pas spécialisé dans } \\
\text { l'activité } k .\end{array}$ & $\begin{array}{l}\text { Le territoire } r \text { n'est pas } \\
\text { spécialisé dans l'activité } k .\end{array}$ & $\begin{array}{l}\text { Le territoire } r \text { n'est pas } \\
\text { spécialisé dans l'activité } k .\end{array}$ & $\begin{array}{l}\text { Le territoire } r \text { est spécialisé } \\
\text { dans l'activité } k .\end{array}$ \\
\hline
\end{tabular}

Tableau 3. Répartition des UGB par type de territoire (NUTS3) en UE.

(Source : Eurostat 2010, traitement des auteurs).

\begin{tabular}{|l|c|c|c|}
\hline \multirow{2}{*}{ Type de production } & \multicolumn{3}{|c|}{ \% des UGB dans les territoires ${ }^{(1)}$} \\
\cline { 2 - 4 } & Sans spécialisation & Mono-spécialisés & 2 spécialités \\
\hline Vaches allaitantes & $31 \%[53 \%]^{(2)}$ & $32 \%[15 \%]$ & $31 \%[24 \%]$ \\
\hline Vaches laitières & $33 \%[59 \%]$ & $27 \%[12 \%]$ & $34 \%[22 \%]$ \\
\hline Porcs & $17 \%[34 \%]$ & $13 \%[5 \%]$ & $65 \%[54 \%]$ \\
\hline Volailles & $31 \%[64 \%]$ & $21 \%[10 \%]$ & $40 \%[19 \%]$ \\
\hline
\end{tabular}

${ }^{(1)}$ La somme de chaque ligne est inférieure à $100 \%$, le solde étant les territoires avec 3 spécialisations. Ces territoires avec 3 spécialités représentent $9 \%$ de la SAU de l'UE et environ $8 \%$ des effectifs des différentes filières animales (vaches laitières, vaches allaitantes, porc et volaille).

${ }^{(2)}[\%]$ : [\% SAU de l'UE accueillant des UGB de l'espèce $\left.k\right]$.

pliqueraient nettement l'étude des résultats en les rendant plus dépendants encore du nombre d'activités animales présente sur un territoire. De plus, les enjeux économiques et environnementaux liés à la spécialisation des territoires concernent principalement les 4 activités animales retenues (vaches laitières, allaitantes, porcs et volailles) ${ }^{3}$.

Pour rendre compte de la localisation des activités d'élevage sur le territoire européen à un niveau spatial relativement fin, nous avons mobilisé la nomenclature des unités territoriales statistiques (NUTS) établie par les services de la Commission européenne dont le niveau 3 découpe le territoire européen en 986 zones géographiques $^{4}$. L'information sur le nombre d'UGB par type de cheptel est issue des données statistiques de 2010 produites par les services d'Eurostat. D'après les données statistiques de 2010, l'UE-28 compte un cheptel global de 135 millions d'UGB dont 37,1 millions d'UGB porcins, 23,4 millions d'UGB vaches laitières, 20,3 millions d'UGB volailles et 9,9 millions d'UGB vaches allaitantes ${ }^{5}$. Ces territoires, définis pour des besoins statistiques non liés aux questions agri- coles, ont des tailles physiques différentes. De même, la quantité d'UGB au niveau national varie fortement d'un type de cheptel à l'autre.

\section{2 / État des spécialisations des territoires de l'UE}

Pour illustrer globalement l'importance des co-localisations des productions animales, nous avons reporté dans le tableau 3 la part des UGB localisée dans des territoires qui n'ont aucune spécialisation, une unique spécialisation et deux spécialisations pour chaque type d'espèce. Autrement dit, nous avons procédé en deux étapes. Nous avons tout d'abord caractérisé la fréquence de chaque espace $\mathrm{k}$ sur chaque territoire $r$ par $S_{r}^{k}>1$. Si la fréquence où $S_{r}^{k}>1$ est égale à $0,1,2$ et 3 alors le territoire $r$ est respectivement avec 0 , une unique, deux spécialisations et avec 3 spécialisations. Ensuite, nous avons déterminé la répartition des UGB de chaque espèce $k$ entre les types de territoire à l'échelle de l'EU. Il apparaît très nettement que les vaches laitières et allaitantes sont réparties de manière homo- gène entre les différents types de territoire. $31 \%$ des UGB de vaches allaitantes sont localisées dans des territoires non spécialisés, $32 \%$ dans des territoires spécialisés, et $31 \%$ dans des territoires bi-spécialisés. À l'inverse, les porcs et, dans une moindre mesures les volailles, sont concentrées dans des territoires avec deux spécialisations. $70 \%$ des porcs sont localisées dans des territoires avec au moins 2 spécialités. Les territoires dans lesquels l'élevage de porcs est la seule production surreprésentée dans des zones à forte densité sont rares. Il s'agit de la Murcie en Espagne mais aussi l'Est du Danemark et la région de Hanovre en Allemagne. Il apparaît également que les productions de lait et de viande bovine sont davantage localisées dans des territoires mono-spécialisés que les productions de porc et de volaille. Pour mieux comprendre cette première tendance observée à l'échelle de l'UE, nous reproduisons la même analyse au niveau des pays.

Pour illustrer l'importance des cospécialisations au sein d'un même pays, nous avons représenté d'une part, la répartition des UGB entre les territoires

\footnotetext{
${ }^{3}$ Parmi les 986 territoires étudiés, 79 territoires sont considérés comme non-spécialisés dans les 4 principales productions animales, mais spécialisés dans la catégorie « autres espèces animales ». Parmi eux, 31 territoires n'ont aucun UGB dans les 4 principales productions animales.

4 En France, le découpage NUTS3 privilégie les départements, soit 99 zones. En comparaison, le nombre de zones est, par exemple, de 139 au Royaume-Uni, 110 en Italie, 66 en Pologne, 59 en Espagne, 51 en Grèce, 44 en Belgique, 42 en Roumanie, 40 aux Pays-Bas, 30 au Portugal, 20 en Hongrie, 11 au Danemark et 1 au Luxembourg. Pour l'Allemagne, nous avons considéré le niveau NUTS2, soit 39 zones contre 412 dans le cas de NUTS3.

${ }^{5}$ Le reste du cheptel est composé des autres bovins (mâles, génisses, veaux), des ovins (brebis laitières, brebis allaitantes, agneaux), des caprins et des équins.
} 
mono-spécialisés et bi-spécialisés par pays (tableau $4 \mathrm{a}$ et $4 \mathrm{~b}$ ), et d'autre part, la fréquence des associations de type de cheptel dans les territoires avec au moins 2 spécialités (tableau 5). Nous concen- trons notre étude sur les 10 plus grands pays producteurs d'animaux de l'UE, et analysons principalement les résultats des principaux pays producteurs de chaque espèce animale. À noter que la
France est le seul pays à être parmi les 4 plus grands producteurs de l'UE dans chacune des 4 espèces animales. En effet, elle détient $33 \%$ des UGB vaches allaitantes (devant l'Espagne et le Royaume-

Tableau 4a. Répartition des UGB par type de territoire (NUTS3) par pays ${ }^{(1)}$.

(Source : Eurostat 2010, traitement des auteurs.)

\begin{tabular}{|l|c|c|c|c|c|c|c|c|}
\hline & \multicolumn{2}{|c|}{ Vaches Allaitantes } & \multicolumn{2}{c|}{ Vaches Laitières } & \multicolumn{2}{|c|}{ Porcs } & \multicolumn{2}{c|}{ Volailles } \\
\hline $\begin{array}{l}\text { Nombre de } \\
\text { spécialisations }\end{array}$ & Une & Deux & Une & Deux & Une & Deux & Une & Deux \\
\hline \multirow{2}{*}{ France } & $\mathbf{7 0 \%}(2)$ & $\mathbf{3 0 \%}$ & $\mathbf{5 2 \%}$ & $\mathbf{4 5 \%}$ & $\mathbf{0}$ & $\mathbf{9 7 \%}$ & $\mathbf{3 5 \%}$ & $\mathbf{6 3} \%$ \\
& {$[55 \%]$} & {$[42 \%]$} & {$[46 \%]$} & {$[45 \%]$} & {$[0 \%]$} & {$[81 \%]$} & {$[31 \%]$} & {$[63 \%]$} \\
\hline \multirow{2}{*}{ Allemagne } & $2 \%$ & $71 \%$ & $\mathbf{7 2 \%}$ & $\mathbf{1 9 \%}$ & $\mathbf{3 6 \%}$ & $\mathbf{5 6 \%}$ & $\mathbf{5 \%}$ & $\mathbf{7 6 \%}$ \\
& {$[2 \%]$} & {$[65 \%]$} & {$[55 \%]$} & {$[26 \%]$} & {$[24 \%]$} & {$[48 \%]$} & {$[3 \%]$} & {$[60 \%]$} \\
\hline \multirow{2}{*}{ Belgique } & $42 \%$ & $52 \%$ & $24 \%$ & $49 \%$ & $41 \%$ & $49 \%$ & $0 \%$ & $76 \%$ \\
& {$[26 \%]$} & {$[66 \%]$} & {$[11 \%]$} & {$[72 \%]$} & {$[43 \%]$} & {$[45 \%]$} & {$[0 \%]$} & {$[67 \%]$} \\
\hline \multirow{2}{*}{ Danemark } & $0 \%$ & $40 \%$ & $0 \%$ & $72 \%$ & $\mathbf{7 3 \%}$ & $\mathbf{2 \%}$ & $0 \%$ & $48 \%$ \\
& {$[0 \%]$} & {$[37 \%]$} & {$[0 \%]$} & {$[68 \%]$} & {$[56 \%]$} & {$[4 \%]$} & {$[0 \%]$} & {$[38 \%]$} \\
\hline \multirow{2}{*}{ Espagne } & $\mathbf{4 8 \%}$ & $\mathbf{6 7 \%}$ & $4 \%$ & $87 \%$ & $\mathbf{6 0 \%}$ & $\mathbf{4 0 \%}$ & $\mathbf{4 1 \%}$ & $\mathbf{5 3 \%}$ \\
& {$[50 \%]$} & {$[47 \%]$} & {$[15 \%]$} & {$[77 \%]$} & {$[67 \%]$} & {$[33 \%]$} & {$[65 \%]$} & {$[30 \%]$} \\
\hline \multirow{2}{*}{ Irlande } & $\mathbf{3 3 \%}$ & $\mathbf{4 0 \%}$ & $0 \%$ & $100 \%$ & $43 \%$ & $53 \%$ & $0 \%$ & $0 \%$ \\
& {$[30 \%]$} & {$[35 \%]$} & {$[0 \%]$} & {$[100 \%]$} & {$[24 \%]$} & {$[61 \%]$} & {$[0 \%]$} & {$[0 \%]$} \\
\hline \multirow{2}{*}{ Italie } & $48 \%$ & $45 \%$ & $\mathbf{3 8 \%}$ & $\mathbf{5 8 \%}$ & $43 \%$ & $53 \%$ & $\mathbf{6 4 \%}$ & $\mathbf{2 7 \%}$ \\
& {$[49 \%]$} & {$[44 \%]$} & {$[30 \%]$} & {$[64 \%]$} & {$[24 \%]$} & {$[61 \%]$} & {$[39 \%]$} & {$[48 \%]$} \\
\hline \multirow{2}{*}{ Pays-Bas } & $5 \%$ & $70 \%$ & $14 \%$ & $66 \%$ & $30 \%$ & $57 \%$ & $22 \%$ & $69 \%$ \\
& {$[4 \%]$} & {$[74 \%]$} & {$[9 \%]$} & {$[72 \%]$} & {$[23 \%]$} & {$[56 \%]$} & {$[9 \%]$} & {$[69 \%]$} \\
\hline \multirow{2}{*}{ Pologne } & $4 \%$ & $79 \%$ & $\mathbf{5 0 \%}$ & $\mathbf{4 4 \%}$ & $\mathbf{5 1 \%}$ & $\mathbf{4 9 \%}$ & $\mathbf{1 2 \%}$ & $\mathbf{8 2 \%}$ \\
& {$[4 \%]$} & {$[83 \%]$} & {$[40 \%]$} & {$[49 \%]$} & {$[51 \%]$} & {$[49 \%]$} & {$[13 \%]$} & {$[77 \%]$} \\
\hline \multirow{2}{*}{ Royaume-Uni } & $\mathbf{3 1 \%}$ & $\mathbf{6 7 \%}$ & $\mathbf{4 6 \%}$ & $\mathbf{4 9 \%}$ & $23 \%$ & $69 \%$ & $15 \%$ & $76 \%$ \\
& {$[49 \%]$} & {$[47 \%]$} & {$[38 \%]$} & {$[53 \%]$} & {$[16 \%]$} & {$[67 \%]$} & {$[14 \%]$} & {$[69 \%]$} \\
\hline
\end{tabular}

(1) Concerne uniquement des territoires avec au moins 1 spécialisation.

${ }^{(2)}$ En gras : les principaux pays producteurs de chaque espèce animale, produisant au moins $8 \%$ des UGB de I'UE.

${ }^{(3)}[\%]$ : [\% SAU du pays accueillant des UGB de l'espèce $\left.k\right]$;

Exemple d'interprétation (ligne 3, colonne 2 et 3) : En France, 70\% des UGB vaches allaitantes appartiennent à des territoires avec cette unique spécialisation, $30 \%$ à des territoires avec 2 spécialisations (dont la production de vaches allaitantes).

Tableau 4b. Part des UGB dans des territoires (NUTS3) spécialisés par pays.

(Source : Eurostat 2010, traitement des auteurs.)

\begin{tabular}{|c|c|c|c|c|}
\hline & Vaches Allaitantes & Vaches Laitières & Porcs & Volailles \\
\hline France & $69 \%^{(1)}[48 \%]^{(2)}$ & $67 \%[41 \%]$ & $69 \%[19 \%]$ & $76 \%$ [35\%] \\
\hline Allemagne & $64 \%[52 \%]$ & $65 \%[52 \%]$ & $64 \%[33 \%]$ & $69 \%[41 \%]$ \\
\hline Belgique & $67 \%[60 \%]$ & $55 \%[52 \%]$ & $89 \%[34 \%]$ & $67 \%[26 \%]$ \\
\hline Danemark & $59 \%[56 \%]$ & $82 \%[59 \%]$ & $39 \%[41 \%]$ & $66 \%[57 \%]$ \\
\hline Espagne & $79 \%[42 \%]$ & $79 \%[24 \%]$ & $71 \%[25 \%]$ & $69 \%[37 \%]$ \\
\hline Irlande & $66 \%[59 \%]$ & $73 \%[44 \%]$ & $86 \%[59 \%]$ & $66 \%[18 \%]$ \\
\hline Italie & $87 \%[58 \%]$ & $66 \%[33 \%]$ & $75 \%[20 \%]$ & $70 \%[34 \%]$ \\
\hline Pays-Bas & $63 \%[59 \%]$ & $75 \%[72 \%]$ & $77 \%[28 \%]$ & $58 \%[36 \%]$ \\
\hline Pologne & $50 \%$ [35\%] & $30 \%[40 \%]$ & $64 \%[40 \%]$ & $69 \%[45 \%]$ \\
\hline Royaume-Uni & $58 \%[48 \%]$ & $83 \%[38 \%]$ & $71 \%[31 \%]$ & $63 \%[28 \%]$ \\
\hline
\end{tabular}

${ }^{(1)}$ En gras : les principaux pays producteurs de chaque espèce animale, produisant au moins $8 \%$ des UGB de I'UE.

(2) $[\%]$ : [\% SAU du pays].

Exemple d'interprétation (ligne 2, colonne 2) : En France, 69\% des UGB vaches allaitantes sont localisées dans des territoires avec au moins 1 spécialisation dans les vaches allaitantes. 
Uni), $21 \%$ des UGB volailles (largement devant l'Espagne, l'Italie et la Pologne), $15 \%$ des UGB vaches laitières (juste derrière l'Allemagne) et $9 \%$ des UGB porcs (derrière l'Allemagne et l'Espagne) de l'UE.

Pour les tableaux $4 \mathrm{a}$ et $4 \mathrm{~b}$, nous avons tout d'abord identifié pour chaque espèce $k$, les territoires $r$ avec au moins une spécialisation dans l'espèce $k$. Nous avons ensuite déterminé pour chaque pays la part des UGB de l'espèce $k$ entre les territoires ayant comme une unique spécialisation l'espèce $\mathrm{k}$, les territoires ayant deux spécialisations dont l'espèce $\mathrm{k}$ et les autres types de territoire. La France se distingue nettement des autres pays européens en matière d'organisation géographique de ces productions animales. Il existe notamment des différences importantes entre les principaux producteurs de vaches allaitantes, la France, l'Espagne, le Royaume-Uni et l'Irlande. En France, l'élevage allaitant se concentre dans des territoires mono-spécialisés, contrairement aux autres pays (tableaux $4 \mathrm{a}$ et $4 \mathrm{~b}$ ). En France, $70 \%$ des UGB vaches allaitantes sont localisés dans des territoires mono-spécialisés, contre seulement $48 \%$ en Espagne, $33 \%$ en Irlande et $31 \%$ au Royaume-Uni. Ces élevages étant très dépendants des aides directes, les différences observées entre les pays s'expliquent, en partie, par les différentes modalités d'application du découplage des aides suite à la réforme de la PAC de 2003. Le Royaume-Uni et l'Irlande ont opté pour un découplage total de la PMTVA, ce qui contribue à sa localisation dans les zones où la compétition avec les autres productions, notamment la production laitière, est la moins forte. En France et en Espagne, le découplage de la PMTVA est partiel, ce qui participe, dans une certaine mesure, à l'ancrage de la production bovine allaitante sur le territoire. La rentabilité à l'hectare de cette production étant faible et la compétition avec les autres productions, notamment laitières, étant forte en France, les élevages allaitants français se sont concentrés dans les zones défavorisées dans lesquelles des aides directes sont attribuées dans le cadre du second pilier de la PAC, notamment l'ICHN (Indemnité Compensatoire de Handicaps Naturels) et la PHAE (Prime Herbagère Agro-Environnementale).

Dans les principaux pays producteurs, les vaches laitières et les porcs se concentrent dans des territoires mono-spécialisés. C'est notamment le cas de l'Allemagne, plus grand pays producteur de lait et de porc de l'UE, dont $72 \%$ des UGB vaches laitières et $36 \%$ des UGB porcs sont localisés dans des territoires avec une unique spécialisation. Ceci est également observé dans les autres principaux pays producteurs de porcs (Danemark, Espagne, Pologne). Au Danemark, $73 \%$ des UGB porcs sont localisés dans des territoires mono-spécialisés, contre $60 \%$ en Espagne, $51 \%$ en Pologne. La France fait encore figure d'exception : elle est le seul pays à n'avoir aucun territoire uniquement spécialisé dans la production porcine.

Dans le tableau 5, nous avons reporté la part des UGB dans des territoires caractérisés par leur type d'association de spécialisations. Nous avons identifié pour chaque pays les territoires avec au moins deux spécialisations dans : i) les vaches laitières et les vaches allaitantes, ii) les vaches laitières et les porcs, et iii) les porcs et les volailles. Le poids en termes d'UGB des associations de productions animales présentes en France diffèrent de celles observées dans les autres pays de l'UE. La France se distingue notamment par le faible poids de l'association Lait/Viande bovine et par le poids non négligeable de l'association Lait/porc sur son territoire. Seulement $12 \%$ des UGB vaches laitières sont localisées dans des territoires avec une spécialisation Lait/Viande bovine, ce qui est le pourcentage le plus faible des pays étudiés, et $15 \%$ sont localisées dans des territoires avec une spécialisation Lait/ Porc (tableau 5). À titre de comparaison, seulement $2 \%$ des territoires allemands associent les productions laitières et porcines sur les mêmes territoires.

Les raisons sont à puiser notamment dans les modalités de mise en œuvre de la politique des quotas laitiers et de la Directive Nitrates (DN). Chaque pays est en mesure de fixer les moyens pour parvenir aux objectifs poursuivis par la DN. Certains pays, comme l'Irlande, les Pays-Bas, le Danemark et l'Allemagne, ont choisi de déclarer l'ensemble de leur territoire en zone vulnérable. La France a choisi de ne classer en zone vulnérable que les territoires dans lesquels l'enjeu nitrate était avéré, comme en Bretagne. Pour toutes ces zones vulnérables européennes, le plafond maximal d'azote organique épandable est fixé à $170 \mathrm{kgN} / \mathrm{ha}$. Cependant, la mise en œuvre de la DN présente de grandes différences entre les

Tableau 5. Fréquence des associations de type de cheptel dans les territoires comportant au moins deux spécialités. (Source : Eurostat 2010, traitement des auteurs.)

\begin{tabular}{|c|c|c|c|c|c|c|c|c|c|}
\hline & \multicolumn{3}{|c|}{$\begin{array}{l}\text { Vaches Laitières (VL) et } \\
\text { Vaches Allaitantes (VA) }\end{array}$} & \multicolumn{3}{|c|}{ Vaches Laitières et Porcs } & \multicolumn{3}{|c|}{ Porcs et Volailles } \\
\hline & \multirow{2}{*}{$\%$ SAU } & \multicolumn{2}{|c|}{$\%$ UGB } & \multirow{2}{*}{$\%$ SAU } & \multicolumn{2}{|c|}{$\%$ UGB } & \multirow{2}{*}{$\%$ SAU } & \multicolumn{2}{|c|}{$\%$ UGB } \\
\hline & & VA & VL & & VL & Porcs & & Porcs & Volailles \\
\hline France & $12^{(1)}$ & 13 & 12 & 7 & 15 & 16 & 11 & 53 & 35 \\
\hline Allemagne & 23 & 33 & 18 & 2 & 2 & 2 & 17 & 35 & 47 \\
\hline Belgique & 42 & 38 & 31 & 4 & 10 & 8 & 20 & 52 & 62 \\
\hline Danemark & 37 & 48 & 46 & 0 & 0 & 0 & 16 & 10 & 10 \\
\hline Espagne & 15 & 37 & 69 & 3 & 6 & 7 & 5 & 22 & 31 \\
\hline Irlande & 12 & 14 & 16 & 33 & 57 & 40 & 18 & 27 & 66 \\
\hline Italie & 14 & 20 & 15 & 6 & 22 & 28 & 6 & 6 & 8 \\
\hline Pays-Bas & 48 & 50 & 51 & 11 & 17 & 18 & 7 & 33 & 29 \\
\hline Pologne & 16 & 23 & 20 & 0 & 0 & 0 & 14 & 25 & 25 \\
\hline Royaume-Uni & 14 & 29 & 30 & 4 & 5 & 5 & 17 & 45 & 39 \\
\hline UE & 19 & 23 & 25 & 8 & 12 & 10 & 14 & 29 & 31 \\
\hline
\end{tabular}

(1) [\% SAU du pays accueillant des UGB des espèces $k]$.

Exemple d'interprétation (ligne1, colonne 4) : En France, 12\% des UGB vaches laitières sont localisées dans des territoires avec la double spécialisation vaches laitières/vaches allaitantes. 
pays, et notamment concernant les normes de production d'azote par les animaux, la durée réglementaire minimale de stockage des lisiers, la mise en place des programmes d'actions, la possibilité de tenir compte de l'élimination ex post des matières organiques dans le bilan azote de la ferme, etc. Ces différentes modalités d'application permettent un chargement à l'hectare très différent d'un État à l'autre. En France, des élevages de porcs dépassant le plafond maximal d'azote peuvent éliminer leur surplus $\mathrm{d}$ 'azote à $\mathrm{l}$ 'aide de stations de traitement (subventionnés par les autorités publiques) et/ou l'exporter vers des terres déficitaires en azote (Letort et Temesgen 2014). Cette mise en œuvre de la DN n'encourage pas les éleveurs porcins à lier leurs productions animales au sol6. La mise en place de la DN est très différente dans les autres grands pays producteurs de porcs (Le Goffe 2013). Le système danois impose depuis 2002 des plafonds d'épandage d'azote des effluents d'élevage plus sévères pour les exploitations de grandes cultures, de porcs et de volailles (140 kg/ha), et l'éleveur n'ayant pas suffisamment de terres en propriété ou en location pour respecter la règle peut établir un contrat d'épandage avec ses voisins. Dans ce cas, les éleveurs rémunèrent les preneurs de lisiers à environ un euro par kilo d'azote ${ }^{7}$. Ce type d'accords existe dans d'autres pays européens (de manière plus ou moins informelle) mais est particulièrement développé et encadré au Danemark. De même, certains länder allemands (SchleswigHolstein) doivent respecter une balance agronomique par parcelle incitant ainsi les éleveurs à réguler leur apport d'azote organique et minéral en fonction du besoin des plantes.

Simultanément, la gestion administrative française des quotas laitiers à l'échelle de chaque département, puis au niveau des bassins de production, a contribué à figer la production laitière sur le territoire. Contrairement à d'autres pays européens, la réglementation de la France a empêché l'échange de quotas laitiers entre les différents bassins de production français. La combinaison des modalités d'application des politiques de quotas laitiers et de la DN ont permis le développement simultané en Bretagne de la production de lait à côté d'autres filières animales. Cependant, il est probable que la fin des quotas laitiers renforce la compétition entre le porc et le lait, et notamment pour les terres d'épandage.
De même, à certaines périodes, comme en 2012, la hausse des prix des céréales a engendré des redistributions territoriales au détriment des activités d'élevage (Gaigné 2015). L'activité laitière a reculé dans certaines zones de polycultureélevage françaises comme en PoitouCharentes. Ce phénomène spatial est amplifié par la mise en place d'un marché libre des quotas laitiers ${ }^{8}$. En Allemagne, la spécialisation croissante des territoires dans la production de lait a été permise dès les années 2000 en raison notamment de la possibilité d'échanger des quotas au sein de bourses régionales dont le périmètre a été progressivement élargi au cours du temps. La production a légèrement diminué dans les zones intermédiaires, moins denses en production laitière, où la concurrence avec les productions végétales est plus forte (Roguet et al 2015). Depuis 2005, les länder du nord (Basse-Saxe, SchleswigHolstein) enregistrent de fortes augmentations de production laitière (près de $20 \%$ ) tandis que la production n'augmente que légèrement en Bavière $(+7 \%)$.

Au contraire, quelques associations de productions animales sont très fréquentes à l'échelle de l'UE. C'est le cas de l'association volaille-porc. En France, $35 \%$ des UGB volailles, $47 \%$ en Allemagne, $31 \%$ en Espagne et $25 \%$ en Pologne sont localisés dans des territoires spécialisés à la fois en porcs et volailles. Les liens qui unissent les productions de granivores (porcs et volailles) au territoire ne sont pas, du fait du type d'alimentation (monogastrique), de même nature que ceux observés dans le secteur des ruminants. Une alimentation principalement achetée à l'extérieur de l'exploitation et non basée sur les productions fourragères, conduit, de facto, à ce que ces productions soient géographiquement plus concentrées. À l'échelle européenne, les productions porcines et avicoles tendent souvent à se concentrer sur les mêmes territoires, même à un niveau géographique relativement fin quand bien même ces deux productions sont rarement pratiquées au sein des mêmes exploitations pour des raisons sanitaires notamment. Leur concentration géographique et co-localisation peuvent s'expliquer par des caractéristiques communes. L'alimentation des porcs et volailles, à base de céréales (70-75\%) et de sources protéiques (soja notamment), se fait principalement à partir d'aliments achetés à (en porc) ou fournis par (en aviculture) des fabricants d'aliments industriels (même si une partie des exploitations le fabrique à la ferme). L'existence de cette co-localisation tend à montrer que les économies d'agglomération que procurent la concentration des productions et plus largement des filières animales dans des régions (s'étant) dotées d'avantages comparatifs l'emportent sur les surcoûts générés par la concurrence sur les facteurs, notamment sur le travail et le foncier. Ces productions ont ainsi pu se développer indépendamment du foncier disponible, seules ou en complément de revenu d'une activité d'élevage herbivore (par exemple en 2010,23\% des élevages de porcs et $17 \%$ des porcs se trouvent dans des exploitations associant des porcs et des vaches laitières) ou céréalière (en 2010, toujours, $19 \%$ des élevages de porcs et $11 \%$ des porcs se trouvent dans des exploitations de polycultureélevage). Les filières porcine et avicole sont aussi très structurées verticalement, en porc par les groupements de producteurs dont certains ont développé leur activité à l'amont et l'aval, en aviculture par l'intégration.

Nous complétons notre analyse en étudiant l'évolution en termes d'organisation du territoire des principaux pays producteurs de l'UE, à savoir l'Allemagne, l'Espagne et la France. Pour cela, nous recalculons les indices de spécialisation et la répartition des UGB par type de territoire pour l'année 2003 afin d'évaluer les évolutions entre 2003 et 2010 .

L'Espagne, deuxième détenteur de porcs, de volailles et de vaches allaitantes de l'UE en 2010, a eu tendance à fortement spécialiser ces territoires dans une production unique, principalement pour les productions avicoles et porcines. En effet, $45 \%$ des UGB porcs étaient localisées dans des territoires monospécialisés en production porcine en 2003, contre $60 \%$ en 2010 , soit une hausse de $33 \%$. De même pour la production de volailles, $24 \%$ des UGB volailles étaient localisées dans des territoires mono-spécialisés en volailles en 2003, contre $41 \%$ en 2010 . Cette évolution s'inscrit dans un contexte d'augmentation du nombre d'UGB porcs $(+12 \%)$ et d'UGB volailles $(+15 \%)$ sur la période considérée. La croissance de la production de porcs et de volailles s'est donc concentrée dans les territoires monospécialisés.

\footnotetext{
${ }^{6}$ À noter que depuis 2012, période postérieure à notre période d'étude, la France a également mis en place un solde de la Balance Globale Azotée (BGA) à

l'échelle de l'exploitation limitée à $50 \mathrm{kgN} /$ ha dans des Zones d'Actions Renforcées (ZAR) très vulnérables à la pollution par les nitrates.

${ }^{7}$ Par comparaison, le coût moyen du traitement du lisier est estimé entre 3 et $6 €$ par kilo d'azote (Téffène 2002).

${ }^{8}$ Le cas du Royaume-Uni est révélateur. Favorisé par un marché libre des quotas, ce mouvement de spécialisation y est ancien puisqu'il avait débuté dès les années quatre-vingt-dix. Il avait alors surtout profité à l'Irlande du Nord dont la production laitière avait augmenté de $40 \%$ (contre - $10 \%$ pour l'Angleterre, et $+10 \%$ pour le Pays de Galles et l'Ecosse).
} 
L'Allemagne, premier détenteur de vaches laitières et de porcs de l'UE en 2010, a également tendance à spécialiser ses territoires dans sa production dominante. $64 \%$ des UGB vaches laitières ${ }^{9}$ étaient localisés dans des territoires monospécialisés en lait en 2003, contre $72 \%$ en 2010 , soit une hausse de $13 \%$. De même pour la production porcine, 29\% des UGB porcs étaient localisés dans des territoires mono-spécialisés en porcs en 2003 , contre $36 \%$ en 2010 , soit une évolution de $24 \%$. Contrairement à l'Espagne, cette évolution ne s'accompagne pas d'une augmentation significative du nombre d'UGB. La croissance des territoires mono-spécialisés s'est donc effectuée au détriment des autres types de territoire.

Enfin, la France, premier détenteur de volailles et de vaches allaitantes, deuxième détenteur de vaches laitières et quatrième détenteur de porcs de l'UE en 2010, n'a pas subi les mêmes évolutions que l'Allemagne et l'Espagne. Mis à part les UGB vaches allaitantes qui se concentrent davantage dans des territoires mono-spécialisés dans cette production $(+23 \%)$, la répartition des autres productions animales entre les différents types de territoires n'a pas évolué significativement entre 2003 et 2010 . De même, le nombre d'UGB est resté relativement stable sur cette période.

\section{3 / Co-spécialisation et densi- tés animales}

Bien que ces territoires peuvent avoir des quantités d'UGB comparables, ils différent fortement par leurs densités animales, exprimées en UGB par hectare de SAU ${ }^{10}$. A l'échelle de l'UE-28, la densité moyenne s'élève à 0,8 UGB totaux par hectare de SAU. Une première approximation révèle que la quantité
d'UGB croît en moyenne avec la quantité de SAU. Quand la SAU double, la quantité d'UGB augmente en moyenne de $86 \%$. Cependant, au sein des pays, des territoires se singularisent par des niveaux élevés de densité animale par hectare de SAU (cf. cartes représentant les territoires de l'UE avec les plus fortes densités animales dans Roguet et al 2015). C'est le cas des Pays-Bas et la Flandre belge mais aussi le nord-ouest de l'Allemagne, l'ouest de la France et le nord de l'Italie (plaine du Pô). Le chargement peut atteindre 11,7 UGB/ha dans le sud-est du Brabant du nord aux PaysBas, et 10,4 UGB/ha dans l'arrondissement de Tielt dans les Flandres. De même, dans le canton de Landivisiau dans le Finistère en Bretagne, la densité animale peut monter localement à des valeurs proches de celles observées aux PaysBas et en Belgique.

Ces territoires très denses en animaux d'élevage sont souvent caractérisés par une diversité de leurs activités d'élevage, comme c'est le cas en Bretagne. On peut donc penser a priori que les territoires où coexistent plusieurs productions animales ont des niveaux plus élevés de densité, renforçant ainsi les impacts négatifs de l'élevage sur l'environnement. Dans ce cas, dans les territoires caractérisés par ces co-spécialisations des productions animales, les effets positifs liés aux économies de co-agglomération seraient supérieurs aux effets négatifs liés à la réglementation environnementale et à la concurrence entre les exploitations pour le foncier et le travail. Des statistiques descriptives de ces territoires sont reportées dans le tableau 6 et semblent confirmer les liens entre la spécialisation et la densité animale. Il faut être prudent cependant sur le lien entre densité animale et impacts environnementaux. Evidemment, comme cela est très bien rappelé dans Peyraud et al (2012), un même niveau de pression azotée peut conduire à des impacts environnementaux différents selon la sensibilité du milieu (climat, type de sol...) et sa capacité à valoriser ou éliminer l'azote apporté par les animaux (modes d'occupation des sols).

Pour mieux identifier les écarts significatifs de densité entre les différents types de territoires, d'associations de productions animales et de pays, nous avons procédé à une analyse économétrique. Les estimations sont réalisées sur 4 échantillons différents, les territoires produisant du porc, des volailles, du lait, et de la viande bovine. Les données utilisées sont les mêmes que pour les analyses précédentes, et sont donc issues des données statistiques de 2010 produites par Eurostat sur tous les pays de l'UE découpés en NUTS3. La structure générale du modèle peut s'exprimer sous la forme suivante :

$$
Y_{i}^{k}=\beta^{k}+\alpha_{1}^{k} D C_{1}+\ldots+\alpha_{S}^{k} D C_{S}+\gamma_{1}^{k} D P_{1}+\ldots+\gamma_{R}^{k} D P_{R}+\varepsilon_{i}^{k}
$$

La variable $Y_{i}^{k}$ correspond à la densité en UGB par hectare de SAU de l'espèce animale $k$ (avec $k=$ porc, volaille, vache laitière, vache allaitante) sur le territoire $i(i=1, \ldots, I)$. Le paramètre $\beta^{k}$ est la constante du modèle. Nous introduisons autant de variables indicatrices que de combinaisons possibles de productions animales (moins une combinaison de référence pour éviter les problèmes de colinéarité). La variable $D C_{s}$ prend la valeur 1 si la combinaison $s$ est présente dans le territoire $i$, et 0 sinon. Les coefficients $\alpha_{1}^{k}, \ldots, \alpha_{s}^{k}$ représentent alors l'effet de chaque combinaison $s(s=1, \ldots, S)$ sur la densité de l'espèce animale $k$. De même, nous introduisons autant de variables indicatrices que de pays (moins un pays de référence). La variable $D P_{r}$ prend la valeur 1 si le territoire $i$ appartient au pays $r$ et 0 sinon. Les coefficients $\gamma_{1}^{k}, \ldots, \gamma_{R}^{k}$ représentent alors l'effet de chaque pays $r(r=1, \ldots, R)$ sur la densité de l'espèce animale $k$. Le terme $\varepsilon_{i}^{k}$ correspond au terme d'erreur du modèle.

Tableau 6. Diversité des spécialisations dans le Top 200 des unités NUTS3 (UGB totales).

(Source : Eurostat 2010, traitement des auteurs).

\begin{tabular}{|c|c|c|c|c|c|c|c|c|c|c|}
\hline \multirow[b]{3}{*}{ Vaches Laitières } & \multicolumn{2}{|c|}{$\begin{array}{c}\text { Territoires } \\
\text { mono-spécialisés } \\
(n=93)\end{array}$} & \multicolumn{8}{|c|}{$\begin{array}{c}\text { Territoires avec } \\
\text { deux spécialisations }(n=91)\end{array}$} \\
\hline & \multirow{2}{*}{\begin{tabular}{|c|} 
Nombre \\
33 \\
\end{tabular}} & \multirow{2}{*}{$\begin{array}{c}\begin{array}{c}\text { Densité } \\
\text { (UGB/ha) }\end{array} \\
1,18 \\
\end{array}$} & \multirow{2}{*}{$\begin{array}{c}\text { Nombre } \\
\mathbf{5 0} \\
\end{array}$} & \multirow{2}{*}{$\begin{array}{c}\begin{array}{c}\text { Densité } \\
\text { (UGB/ha) }\end{array} \\
1,53\end{array}$} & \multicolumn{2}{|c|}{$\begin{array}{c}\text { Vaches } \\
\text { Allaitantes }\end{array}$} & \multicolumn{2}{|c|}{ Porcs } & \multicolumn{2}{|c|}{ Volailles } \\
\hline & & & & & 28 & 1,2 & 16 & 1,9 & 6 & 1,6 \\
\hline Vaches Allaitantes & 18 & 0,74 & 41 & 1,14 & & - & 7 & 1,0 & 6 & 0,6 \\
\hline Porcs & 26 & 2,15 & 51 & 2,52 & & & & - & 28 & 3,2 \\
\hline Volailles & 16 & 1,90 & 40 & 2,60 & & & & & - & - \\
\hline
\end{tabular}

\footnotetext{
${ }^{9}$ Pourcentage calculé par rapport aux UGB vaches laitières appartenant à un territoire avec au moins 1 spécialisation en lait (tableau 3a).

${ }^{10}$ La mesure de la densité animale exprimée en UGB par hectare de SAU ne traduit pas toujours la réalité des densités animales des élevages. En effet, dans les territoires où une part très importante de la SAU n'est pas valorisée par l'élevage, les effectifs animaux sont dilués avec des terres utilisées pour d'autres usages (céréales, fruits, vignes...), alors que les élevages de ces territoires peuvent avoir des densités animales très élevées.
} 
Les résultats de l'estimation sont présentés dans le tableau 7. Tous les résultats obtenus sont à comparer au territoire de référence. Par exemple, dans le cas des territoires produisant du porc (colonne 1), le type de territoire de référence regroupe les territoires français mono-spécialisé en porcs. L'interprétation se fait de cette manière : les territoires bi-spécialisés porc/volaille ont une densité animale moyenne supérieure de $0,33 \mathrm{UGB} / \mathrm{ha}$ à la densité moyenne observée sur les territoires français mono-spécialisés en porcs.

Les résultats de l'analyse révèlent qu'il existe des écarts significatifs de densité selon les pays et la nature des spécialisations. Parmi les mono-spécialisations, celle dans le porc génère des niveaux de densités élevés. Cela reste vrai, quel que soit le pays. Par ailleurs, il n'y pas d'écart significatif en moyenne de densité entre des territoires mono-spécialisés dans la volaille et ceux mono-spécialisés dans le lait. Les territoires uniquement spécialisés dans les vaches allaitantes ont en moyenne des densités animales plus faibles. Il apparaît également que les territoires avec deux spécialisations lait et porc génèrent des niveaux de densités bien plus élevés que les territoires avec une unique spécialisation dans le lait, mais non significativement différents des territoires avec une unique spécialisation dans les porcs. Cette association lait et porc sur un même territoire est observée en France, notamment en Bretagne. Il est de plus en plus fréquent dans ces territoires, que les exploitations porcines expor- tent leurs effluents excédentaires sur des terres voisines déficitaires en azote appartenant aux exploitations laitières ${ }^{11}$. La disponibilité locale de terre pour l'épandage des effluents d'élevage donne ainsi la possibilité aux exploitations porcines de maintenir la taille de leur cheptel tout en sécurisant leur plan d'épandage et en respectant la réglementation environnementale qui plafonne les apports d'azote organique par hectare. Les exploitations laitières y gagnent également, puisqu'elles bénéficient d'apports d'engrais organiques à moindre coût. Ces échanges d'effluents, généré par la mise en œuvre française de la Directive Nitrate, pourrait faciliter le maintien de la co-agglomération de ces productions animales sur un même territoire en France (Conseil des communautés européennes 1991).

Tableau 7. Modèles explicatifs des densités animales exprimées en UGB/ha SAU à l'échelle NUTS3 et coefficients des différentes variables explicatives.

\begin{tabular}{|c|c|c|c|c|}
\hline & UGB Porcs > 0 & UGB Volailles > 0 & UGB VL > 0 & UGB VA > 0 \\
\hline Constante & $+1,23^{* * *}$ & $+0,88^{* * *}$ & $+0,87^{\star * *}$ & $+0,47^{* * *}$ \\
\hline Sans spec & $-0,67^{* * *}$ & $-0,38^{* * *}$ & $-0,33^{*}$ & $-0,03$ \\
\hline Mono-Porcs & référence & $+0,34^{* *}$ & $+0,41^{* *}$ & $+0,81^{* * *}$ \\
\hline Mono-Volailles & $-0,32^{*}$ & référence & 0,03 & $+0,41^{* \star *}$ \\
\hline Mono-VL & $-0,34^{\star * *}$ & $+0,02$ & référence & $+0,42^{\star \star *}$ \\
\hline Mono-VA & $-0,77^{* * *}$ & $-0,43^{* * *}$ & $-0,45^{\star * *}$ & référence \\
\hline $\begin{array}{l}\text { Duo- } \\
\text { Porcs/Volailles }\end{array}$ & $+0,33^{* * *}$ & $+0,68^{\star * *}$ & $+0,70^{* * *}$ & $+1,11^{\star * *}$ \\
\hline Duo-Porcs/VL & $+0,11$ & $+0,45^{* *}$ & $+0,46^{\star *}$ & $+0,87^{* * *}$ \\
\hline Duo-Volailles/VL & $-0,51^{* *}$ & $-0,17$ & $-0,14$ & $+0,28^{*}$ \\
\hline Duo-VL/VA & $-0,73^{* * *}$ & $-0,38^{* * *}$ & $-0,42^{\star * *}$ & $<0,001$ \\
\hline France & référence & référence & référence & référence \\
\hline Allemagne & $+0,22$ & $+0,22$ & $+0,23$ & $+0,22$ \\
\hline Belgique & $+2,17^{* * *}$ & $+2,14^{* * *}$ & $+2,15^{\star * *}$ & $+2,14^{* * *}$ \\
\hline Danemark & $+0,84^{\star \star *}$ & $+0,84^{* * *}$ & $+0,83^{* * *}$ & $+0,71^{* *}$ \\
\hline Espagne & $+0,06$ & $+0,07$ & $+0,14$ & $+0,08$ \\
\hline Irlande & $+0,34$ & $+0,35$ & $+0,36$ & $+0,35$ \\
\hline Italie & $+0,06$ & $+0,03$ & $+0,04$ & $+0,03$ \\
\hline Pays-Bas & $+2,74^{\star \star *}$ & $+2,78^{\star * *}$ & $+2,51^{* * *}$ & $+2,49 * * *$ \\
\hline Pologne & $-0,24^{*}$ & $-0,24$ & $-0,25^{\star \star \star}$ & $-0,23$ \\
\hline Royaume Uni & $+0,02$ & $+0,02$ & $+0,06$ & $+0,05$ \\
\hline $\begin{array}{l}\text { Nombre } \\
\text { d'observations }\end{array}$ & 916 & 934 & 920 & 919 \\
\hline
\end{tabular}

VL : Vaches Laitières ; VA : Vaches Allaitantes.

Non rapporté : autres combinaisons de production.

${ }^{*},{ }^{* *},{ }^{* * *}$ significativement différent de 0 à respectivement, $10 \%, 5 \%$ et $1 \%$.

${ }^{11}$ Les exploitations laitières bretonnes ont en moyenne une pression azotée inférieure au plafond réglementaire de $170 \mathrm{kgN} / \mathrm{ha}($ Aarts et al 2012 , Letort et al 2017). La contrainte d'épandage n'est donc pas un facteur limitant pour les exploitations laitières, contrairement aux exploitations porcines. 
Toutefois, c'est la combinaison de spécialisation porc/volaille qui génère les niveaux de densité les plus élevés. En recourant à des achats d'aliments en provenance d'autres régions/pays et en étant moins directement dépendantes du facteur foncier, les productions avicoles et porcines autorisent des niveaux d'intensification supérieurs aux productions d'herbivores. De plus, le développement des techniques de séparation des phases et déshydratation des effluents d'élevage pourrait accroître la co-localisation des différentes productions animales en facilitant le transport des excès d'azote et de phosphore. Ces équipements étant potentiellement transfilières, ils pourront renforcer la concentration globale des productions animales sans avoir d'effet sur le poids relatif des différentes filières animales. En théorie, ces technologies de traitement des effluents et de transport des matières organique (stratégie souvent adoptée dans les pays du Nord de l'Europe) pourraient contribuer à accentuer la dissociation spatiale des productions animales et végétales et à une association encore plus forte des différentes productions animales à l'échelle des territoires.

\section{Conclusion}

À l'échelle de l'exploitation, les élevages se spécialisent de plus en plus dans une unique production animale. À l'échelle du territoire NUTS3, cette étude met en avant que de nombreux territoires des grands pays producteurs de l'UE tendent également à être spécialisés (selon la définition retenue dans l'étude) dans une unique production animale. Cette organisation des filières suggère que les gains à la co-agglomération de différentes productions animales sont relativement faibles. Une évaluation rigoureuse des gains et des coûts générés par une co-localisation est toutefois nécessaire afin de confirmer ces premières conclusions ou apporter des éclairages différents.

Nos résultats montrent également que les territoires les plus denses sont souvent associés à la production porcine, qu'elle soit associée ou non à une autre production animale. L'association porcs-volailles engendre des densités particulièrement élevés. Dans les régions productrices de lait, la co-spécialisation lait-porc implique également des densités plus élevées que la mono-spécialisation lait. Si les gains économiques à la co-spécialisation de ces différentes productions sont faibles, une séparation géographique de ces productions permettrait de réduire les densités animales (et les excès d'azote et de phosphore) dans certains territoires tout en maintenant la compétitivité des filières.

La France se distingue des autres pays producteurs d'animaux. Outre la perte de compétitivité dans les différents secteurs d'élevages sur les marchés internationaux, les modalités d'application en France des politiques agricoles, environnementales et même foncières semblent agir comme des freins au processus de monospécialisation des territoires d'élevage dans les filières avicoles, laitières et/ou porcines. Cependant, même si d'autres formes d'organisation spatiale des filières économiquement et environnementalement plus efficaces sont possibles en France, rien ne nous garantit que les différents acteurs d'une filière soient en mesure d'adopter individuellement ces nouveaux modes d'organisation. En effet, les processus d'agglomération engendrent un effet de verrouillage entraînant une grande inertie dans la répartition géographique des secteurs d'activité et la spécialisation des territoires (Krugman 1991). Nous pouvons identifier deux grands freins au changement d'organisation géographique des différentes filières animales. Tout d'abord, les coûts de coordination entre les différents acteurs d'une filière pour changer de trajectoire sont élevés (plus les acteurs sont nombreux, ce qui est le cas en agriculture, plus ces coûts sont substantiels). Ensuite, les coûts liés au changement d'organisation géographique et industrielle se manifestent avant les gains, dont l'ampleur est incertaine, ce qui compliquent fortement toute modification des équilibres territoriaux.

\section{Références}

Aarts F., Béline F., Bockstaller C., Bourblanc M., Delaby L., Dourmad J.Y., Gaigné C., 2012. Les flux d'azote liés aux élevages, réduire les pertes, rétablir les équilibres : synthèse de l'expertise scientifique collective réalisée par l'INRA à la demande des ministères en charge de l'Agriculture et de l'Écologie. Chatellier V., Gaigné C., 2012. Les logiques économiques de la spécialisation productive du territoire agricole français. Innov. Agronom., 22, 185-203.

Conseil des communautés européennes, 1991. Directive 91/676/CEE du Conseil, du 12 décembre 1991, concernant la protection des eaux contre la pollution par les nitrates à partir de sources agricoles, Journal officiel $n^{\circ} \mathrm{L} 375 \mathrm{du}$ $31 / 12 / 1991,1-8$

Cronon W., 1991. Nature's metropolis: Chicago and the Great West. New York, Norton \& Company.

Fujita M., Thisse J.F., 2002. Economics of agglomeration: cities, industrial location, and regional growth. Cambridge, Cambridge University Press.

Gaigné C., 2015. Localisation de la production alimentaire. L'alimentation à découvert.
Catherine E., Jean F., Bruno L. CNRS Éditions, 243-244.

Gaigné C., Ben Arfa N., 2011. Environnement et concentration géographique des productions animales : quels effets sur la compétitivité de l'Ouest de la France ? Focus PSDR3, 1-4.

Gaigné C., Le Gallo J., Larue, S., Schmitt B., 2012. Does regulation of manure land application work against agglomeration economies? theory and evidence from the french hog sector. Am. J. Agr. Econ., 94, 116-132.

Isik M., 2004. Environmental regulation and the spatial structure of the U.S. Dairy sector. Am. J. Agr. Econ., 86, 949-962.

Krugman P., 1991. Increasing returns and economic geography. J. Pol. Econ., 99, 483-499.

Le Goffe P., 2013. La directive nitrates, incompatible avec l'élevage? Le cas de la France et des pays d'Europe du nord (No. hal-01208879).

Letort C., Temesgen C., 2014. Influence of environmental policies on farmland prices in the Bretagne region of France. Rev. Agricult. Environ. Stud., 95-1, 77-109.
Mosnier C., Wieck C., 2012. Dynamiques régionales de la production laitière : France Allemagne - Royaume-Uni. Econ. Rurale, 132146.

Peyraud J.L., Cellier P., Donnars C., 2012. Rapport d'expertise scientifique collective, INRA, Paris, France, 516p.

Roe B., Irwin E.G., Sharp J.S., 2002. Pigs in Space: Modeling the Spatial Structure of $\mathrm{Hog}$ Production in Traditional and Nontraditional Production Regions. Am. J. Agr. Econ., 84, 259-278

Roguet C., Gaigné C., Chatellier V., Cariou S., Carlier M., Chenu, R., Perrot C., 2015. Spécialisation territoriale et concentration des productions animales européennes : état des lieux et facteurs explicatifs. INRA Prod. Anim., 28, 5-22.

Teffène $\mathrm{O}$, , 2002. Les stations de traitement des effluents porcins, estimation des coûts et conséquences économiques, ITP, Collection «Études économiques » 25,4 . 


\title{
Résumé
}

Si les différents gains associés à la concentration spatiale au sein d'une même filière animale sont relativement bien identifiés, les combinaisons des différentes filières animales au niveau des territoires en Europe sont rarement étudiées. Cet article vise $i$ ) à réaliser un état des lieux de la co-localisation des différentes filières animales au sein des territoires européens, à partir d'un indicateur statistique mesurant la spécialisation des territoires, et ii) à discuter le lien entre la nature des spécialisations et les densités animales à travers une analyse économétrique. À l'échelle du territoire, cette étude met en avant que de nombreux territoires des grands pays producteurs de l'UE sont spécialisés dans une unique production animale. Ceci suggère que les gains à la co-agglomération de différentes productions animales sont relativement faibles. En France, les modalités d'application des politiques agricoles, environnementales et même foncières semblent agir comme des freins au processus de mono-spécialisation des territoires d'élevage dans les filières avicoles, laitières et porcines.

\begin{abstract}
Co-location of different animal production sectors within European countries: the French exception?

Although the gains from the concentration of animal production have been relatively well identified, we have little knowledge about the combination of different animal production types at the territorial level in Europe. This article is aimed at $i$ ) studying the co-location of different animal production types within European territories, using a statistics indicator for measuring territory specialization, and ii) discussing the link between the nature of these specializations and the animal densities within an econometric analysis. This study highlights that, except for France, the major EU producers tend to specialize their territories in a unique animal production. This suggests that the co-location benefits are relatively low. In France, how agricultural, environmental and land policies are implemented seems to have slowed down the mono-specialization of territories, especially in the poultry, pig and dairy sectors.
\end{abstract}

GAIGNÉ C., LETORT E., 2017. Co-localisation des différentes productions animales en Europe : l'exception française ? INRA Prod. Anim., 30, 219-228. 